\title{
COMMENTARY
}

\section{Antisense Sense in Osteoclasts}

Teun J. de Vries

From the Department of Periodontology, Academic Centre for Dentistry Amsterdam (ACTA), Research Institute MOVE, University of Amsterdam and VU University, Amsterdam, the Netherlands

Osteoclasts are the multinucleated, bone-resorbing cells that differentiate from cells of the monocyte-macrophage lineage. At some stage, cells of this lineage may branch off and become committed to either macrophage or osteoclast differentiation. Little is known which molecular pathways determine such branching of developmental fates. An unexpected finding by $\mathrm{Li}$ et $\mathrm{al}^{1}{ }^{1}$ in the current issue of The American Journal of Pathology (AJP), was the observation that osteoclasts produce high levels of zinc-finger transcription factor Wilms' tumor-1 (WT1) antisense RNA, whereas earlier stages in differentiation and also macrophagecommitted cells make the sense variant. This makes WTI a putative osteoclast differentiation inhibitor gene, a gatekeeper that prohibits osteoclast formation. Ultimate absence of WT1 protein due to this high level of antisense RNA in differentiating osteoclast precursors could pave the road for osteoclast differentiation transcription factors such as nuclear factor of activated $\mathrm{T}$ cells, cytoplasmic 1 , leading to osteoclast differentiation commitment.

\section{The Emerging Sense of Antisense}

The magnitude of the contribution of antisense regulation of translation of proteins has only been recognized recently. Two classes of noncoding RNAs are recognized, those with a short length ( $<200$ nucleotides) such as siRNAs and miRNAs or those with a long length, the long noncoding RNAs $(>200$ nucleotides). In a recent review, it was estimated that approximately $30 \%$ of annotated transcripts in humans are antisense transcripts. ${ }^{2}$ Yelin et $\mathrm{al}^{3}$ have identified no less than 2667 human genes that are transcribed in sense as well as antisense directions. Also in the field of osteoclast differentiation, certain microRNAs were shown to particularly interfere with differentiation, as recently summarized by Van der Eerden. ${ }^{4}$ To discriminate between sense and antisense RNAs, one has to specifically make use of techniques that allow this detection. $\mathrm{Li}$ at al ${ }^{1}$ have used three techniques to demonstrate the detection and function of antisense molecules. cDNA was generated with specific sense primers instead of random hexamers. Detection of antisense RNAs such as the WT1 antisense RNA in tissue sections was performed by applying a sense RNA probe rather than a DNA probe. Finally, cells were transfected with specifically the antisense WT1 to study the biological function of antisense WT1 RNA.

\section{WT1 and Hematopoiesis}

WT1 was originally described as a tumor suppressor gene. Mutations of the WTI gene that disrupt its function were associated particularly with tumors of the urogenital tract, including the kidney-associated Wilms' tumor. Mice lacking WT-1 die prematurely in utero due to malfunctioning kidneys. ${ }^{5}$ Evidence for the role of WTl in hematopoiesis and in particular the development of the myeloid lineage, to which osteoclast precursors belong, came from WT1 transfection studies of U937 and HL60 cell lines, where the percentage of $\mathrm{CD}_{11 \mathrm{~b}^{+}}$cells, as a marker of this lineage greatly increased after transfection. This evidence formed the starting point for the present study by Li et al. ${ }^{1}$ During differentiation, WTI caused growth arrest. WT1 was detectable in granulocytes, monocytes, and B lymphocytes. ${ }^{6}$ These results were confirmed by making use of embryonic stem cells from $\mathrm{Wt} 1$ deficient mice that displayed reduced hematopoiesis. ${ }^{7}$

\section{WT1 Antisense and 0steoclast Differentiation}

WT1 expression in cells of the monocyte-macrophage lineage served as a starting point in the study of $\mathrm{Li}$ et al. ${ }^{1}$

\footnotetext{
Accepted for publication June 1, 2016.

Supported in part by departmental funding.

Disclosures: None declared.

Address correspondence to Teun J. de Vries, Ph.D., Department of Periodontology, University of Amsterdam and VU University, Gustav Mahlerlaan 3004, Amsterdam 1081 LA, The Netherlands. E-mail: teun.devries@ acta.nl.
} 
Unexpectedly, mouse RAW-D cells and rat bone marrow cells lost WT-1 protein expression during osteoclast differentiation. Osteoclasts play an important physiological role in tooth eruption by eating away the bone that overlies the coronal part of erupting teeth. In tissue sections of jaws from rat pups with un-erupted teeth, only moderate RNA in situ hybridization signals were observed in osteoclasts with an antisense RNA probe. Intriguingly, however, very strong staining was observed with the negative control sense probe, with which abundant antisense RNA was detected in osteoclasts. From this point on, the authors deserve credits for their perseverance. Surely, many researchers would have, at this point, ignored the result, or used another negative control, or just discarded the in situ hybridization results. Li et $\mathrm{al}^{1}$ instead strengthened their findings by other studies in the field of WTI and the functioning of the kidney, where antisense RNA was detected. They confirmed the presence of antisense RNA in young jaws by Northern blot analysis, showing exclusively large RNA sizes with the sense probe and no RNA with the antisense probe. These data were further confirmed by cDNA synthesis using sense primers. Finally, by transfecting the RAW cell line with antisense RNA, the functionality of antisense RNA was established: osteoclast formation was fivefold induced by antisense WT1, coinciding (only) with increased Ctsk (mRNA for osteoclast enzyme Cathepsin K) mRNA expression.

\section{WT1: A Master Gene Determining Macrophage or Osteoclast Differentiation?}

Although the study by $\mathrm{Li}$ et $\mathrm{al}^{1}$ supports the idea that expression of WT1 in precursors of both macrophages and osteoclasts determines differentiational fate, more conclusive experiments should confirm this further. First of all it should be established whether overexpression of WTI leads to macrophage differentiation instead of osteoclast differentiation, even in the presence of macrophage colonystimulating factor and receptor activator of NF- $\mathrm{KB}$ ligand. Secondly, it should be established what the sense/antisense characteristics of WT1 are in genuine, defined, and sorted bone marrow osteoclast precursors.

Li et al ${ }^{1}$ have only studied WT1 expression in a cell line, in bone marrow, and in the spleen, where contribution of lymphocyte and granulocyte WTI expression may have contributed to the observed expression. The system of osteoclastogenesis insensitive precursors that are sensitized when seeded on bone surfaces would be an interesting model to investigate the putative shifts in sense/antisense transcripts. ${ }^{8}$ Fine-tuning of transcriptional changes should be performed by analyzing genes of such osteoclast differentiation cultures over time, using quantitative PCR approaches, where osteoclastogenesis gene expression such as nuclear factor of activated $\mathrm{T}$ cells, cytoplasmic 1 and anti-osteoclastogenesis signals such as V-maf musculoaponeurotic fibrosarcoma oncogene homolog B should be

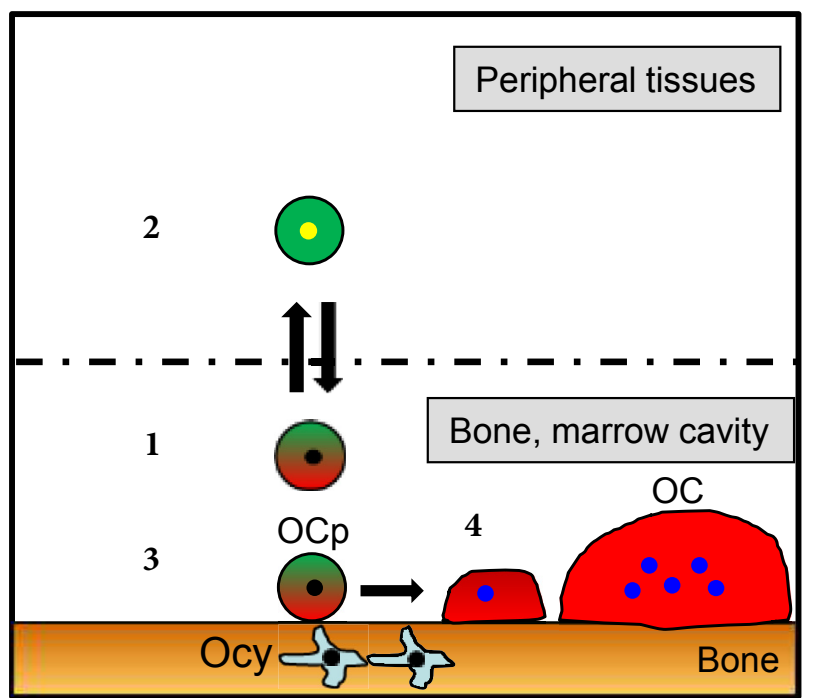

Figure 1 Putative physiological role of WT1 sense and antisense transcripts and WT-1 protein during macrophage and OC differentiation. 1: Bone marrow precursors for macrophages could bear equal levels of sense and antisense WT1 RNA. 2: Macrophages in peripheral tissues express sense WT RNA (green) and express WT-1 protein in the nucleus (yellow), which may protect these cells against $O C$ differentiation. 3: In the proper environment, on bone surfaces and in touch with RANKL-expressing Ocys, the balance of WT1 sense and antisense RNA in OCps may shift toward more antisense (red). 4: Consequently, these cells will no longer express $O C$ inhibitory transcription factors such as WT1 and MafB and start expressing osteoclastogenesis favorable transcription factors such as NFATc1 (blue nuclei), on which multinucleated $\mathrm{OCs}$ form. MafB, V-maf musculoaponeurotic fibrosarcoma oncogene homolog B; NFATC1, nuclear factor of activated $\mathrm{T}$ cells, cytoplasmic 1; OC, osteoclast; OCp, osteoclast precursor; Ocy, osteocyte; RANKL, receptor activator of NF- $\kappa B$ ligand; WT1, Wilms' tumor-1.

investigated in parallel. A putative mechanism displaying the role of WT1 in osteoclastogenesis is shown in Figure 1.

Finally, and most importantly, mouse models should be developed to establish the role of WT1 in osteoclast/ macrophage differentiation. Because the total knockout is lethal in utero, ${ }^{5}$ mice overexpressing either WT1 sense or WT1 antisense RNA under the control of the osteoclast specific Cathepsin K promotor should shed light on whether this leads to bone phenotypes with less (sense) or more (antisense) active osteoclasts.

\section{References}

1. Li Y-L, Kukita A, Kyumoto-Nakamura Y, Kukita T: Extremely high expression of antisense RNA for Wilms' tumor 1 in active osteoclasts: suppression of Wilms' tumor 1 protein expression during osteoclastogenesis. Am J Pathol 2016, 186:2317-2325

2. Pelechano V, Steinmetz LM: Gene regulation by antisense transcription. Nat Rev Genet 2013, 14:880-893

3. Yelin R, Dahary D, Sorek R, Levanon EY, Goldstein O, Shoshan A, Diber A, Biton S, Tamir Y, Shosravi R, Nemzer S, Pinner E, Walach S, Bernstein J, Savitsky K, Rotman G: Widespread occurrence of antisense transcription in the human genome. Nat Biotechnol 2003, 21: $379-386$ 
4. van der Eerden BC: MicroRNAs in the Skeleton: cell-restricted or potent intercellular communicators? Arch Biochem Biophys 2014, 561:46-55

5. Kreidberg JA, Sariola H, Loring JM, Maeda M, Pelletier J, Housman D, Jaenisch R: WT-1 is required for early kidney development. Cell 1993, 74:679-691

6. Ellisen LW, Carlesso N, Cheng T, Scadden DT, Haber DA: The Wilms tumor suppressor WT1 directs stage-specific quiescence and differentiation of human hematopoietic progenitor cells. EMBO J 2001, 20:1897-1909
7. Cunningham TJ, Palumbo I, Grosso M, Slater N, Miles CG: WT1 regulates murine hematopoiesis via maintenance of VEGF isoform ratio. Blood 2013, 122:188-192

8. De Vries TJ, Schoenmaker T, Aerts D, Grevers LC, Souza PPC, Nazmi K, van de Wiel MA, Ylstra B, van Lent PJ, Leenen PJM, Everts V: M-CSF priming of osteoclast precursors can cause osteoclastogenesis-insensitivity, which can be prevented and overcome on bone. J Cell Physiol 2015, 230:210-225 